\title{
Intraoperative neuromonitoring for scoliosis surgery: is there an end to justify the means?
}

\author{
Jason Chui, MBChB, MSc, FANZCA, FHKCA, FHKAM • Alana M. Flexman, MD, FRCPC $\odot$
}

Received: 25 September 2020/ Accepted: 4 October 2020/Published online: 6 January 2021

(C) Canadian Anesthesiologists' Society 2021

Neurologic injury to the spinal cord or nerve roots is a dreaded complication following scoliosis surgery. Thankfully, the incidence of neurologic complications has declined significantly over the last thirty years-from as high as $17 \%$ in $1984^{1}$ to as low as $0.39 \%$ in $2016 .^{2}$ Such injuries can occur secondary to acute traction or tethering of the spinal cord during correction of the spinal curvature, or as a result of direct injury during spinal instrumentation. The Stagnara wake-up test was first described in $1973^{3}$ and involves waking the patient up in the middle of surgery to assess intraoperative spinal cord function. This early neuromonitoring technique was popular in the 1980s and 1990s and remains the gold standard method for evaluating the integrity of neurologic structures in scoliosis surgery. It also does not require any additional equipment. Nevertheless, the wake-up test is often challenging to perform safely in practice, and at best provides only a crude assessment of the motor function at a single timepoint during the surgery rather than detecting the time of injury onset. Furthermore, this period of lightened anesthesia may be complicated by loss of airway and intravenous access and disruption of the sterile field.

This editorial is related to MS 20-00034.

J. Chui, MBChB, MSc, FANZCA, FHKCA, FHKAM

Department of Anesthesia \& Perioperative Medicine, University of Western Ontario, London, ON, Canada

A. M. Flexman, MD, FRCPC ( $\square)$

Department of Anesthesiology, Pharmacology and Therapeutics, University of British Columbia, Vancouver, BC, Canada

e-mail: Alana.flexman@ubc.ca

Department of Anesthesiology, St. Paul's Hospital, 1081 Burrard Street, Vancouver, BC V6Z 1Y6, Canada
The use of somatosensory evoked potentials (SSEP) to monitor the function of the dorsal column as a surrogate of the spinal cord integrity was first introduced clinically in the 1980s. Subsequently, motor evoked potentials (MEP) were added in 1990s to further monitor the functional integrity of the corticospinal tract, with improvement in overall diagnostic performance compared with SSEPs alone. ${ }^{4}$ The potential role of sophisticated and evolving neuromonitoring modalities to mitigate neurologic injury is understandably compelling, although high-quality evidence supporting the ability of these neuromonitoring techniques to improve neurologic outcomes is lacking. ${ }^{5}$ Notwithstanding the absence of strong evidence, the potential benefits have led to widespread adoption of neuromonitoring in many parts of the world. Currently, the use of neuromonitoring is at least partially driven by local medicolegal contexts, availability and various economic factors, which has resulted in a wide variety of practices worldwide.

In this issue of the Journal, Nassef et al. reported a retrospective before-and-after study to compare the effect of the Stagnara wake-up test with evoked potentials (EP) monitoring on postoperative neurologic deficits in 547 adolescent patients (10-18 yr old) who underwent scoliosis correction surgery in two Canadian institutions between 2007 and 2014. ${ }^{6}$ Both institutes transitioned their neurologic monitoring technique from the Stagnara wakeup test to EP monitoring around 2009/2010. In their analysis, the authors found that the patients who received intraoperative neuromonitoring were less likely to develop postoperative neurologic deficits compared with those who underwent the Stagnara wake-up test (odds ratio, 0.39; $95 \%$ confidence interval, 0.18 to $0.86 ; P=0.020$ ). The authors concluded multimodal intraoperative neuromonitoring (SSEPs and MEPs) is associated with reduced overall 
incidence of new neurologic deficits, although they were not able to adjust for confounding variables. ${ }^{6}$ Given the paucity of evidence describing the role of neurologic monitoring and neurologic outcomes in spine surgery, the authors should be commended for undertaking this study and sharing their experience. This study represents one of very few cohorts in the literature in which a direct head-tohead outcome comparison was made between these two intraoperative neurologic monitoring techniques in scoliosis surgery.

Although this study adds to the literature and our understanding of the role of intraoperative neuromonitoring, we must interpret these findings in the context of the study limitations. The limited sample size and low event rate of neurologic deficits in this study precluded the possibility of adjusting for potential confounders. This is particularly important as the neuromonitoring modalities were separated in time, and historical confounding is likely. Specifically, it is likely that a consistent secular trend of general outcome improvement in spinal surgery has occurred over the timeframe studied. The shorter operative times and reduced blood loss seen in the more recent EP monitoring group supports the notion of improved surgical and anesthetic practice over time. Another inherent limitation of this retrospective study is the lack of standardized neurologic assessments and the potential for missing data.

The current evidence supporting the use of EP monitoring in scoliosis surgery largely stems from cohort studies reporting high diagnostic test accuracies of EP monitoring in detecting neurologic injury. A recent systematic review of seven studies involving 2,052 patients reported the point estimates of pooled positive and negative predictive values of combined SSEP and MEP in detecting neurologic injury in scoliosis surgery were $12.2 \%$ and $99.8 \%$, respectively. ${ }^{4} \mathrm{~A}$ previous Canadian study from the Children's Hospital of Eastern Ontario reported positive and negative predictive values of $12 \%$ and $98.2 \%$, respectively. ${ }^{7}$ Nevertheless, almost all these studies were retrospective in nature and, most importantly, all these studies suffered from potential misclassification bias. ${ }^{5}$ Misclassification bias may occur when a rescue intervention is typically employed in the event of the intraoperative identification of EP alert, therefore the rescue intervention (i.e., treatment) may alert the outcome before a reference test is applied (i.e., postoperative neurologic examination) in these studies. Given the uncertainty of how outcome is altered by the rescue intervention, the validity of the reported diagnostic accuracies of EP monitoring in detecting neurologic injury in scoliosis surgery remains controversial. To further complicate the issue, other authors have advocated for EP monitoring as a therapeutic tool to improve neurologic outcomes after scoliosis surgery. Numerous reports have highlighted cases of intraoperative neurologic injury that were promptly reversed by an earlier intervention as a result of earlier detection from EP monitoring. ${ }^{8,9}$ Additionally, several large case series have reported an exceedingly low incidence of postoperative neurologic deficits in patients receiving EP monitoring, thereby indirectly supporting a positive therapeutic role for EP monitoring. ${ }^{10-13}$ The actual role for EP monitoring toward improving outcomes remains elusive, as no randomized-controlled study has been performed to show the efficacy of EP monitoring to minimize neurologic injury in scoliosis surgery.

Despite the lack of efficacy and the lack of a robust assessment of diagnostic test accuracy, EP monitoring has already largely replaced the Stagnara wake-up test as the preferred neurologic monitoring technique in scoliosis surgery in Canada, the United States and many other countries. This change was partly driven by the risks and technical difficulties of performing the Stagnara wake-up test, and partly driven by the perceived non-inferiority outcomes of patients being monitored by EP monitoring in retrospective non-randomized studies. A report of the US National Inpatient Sample database found the utilization of EP monitoring in spine surgery had a four-fold increase from 31,762 cases in 2008 to 125,835 cases in $2014 .{ }^{13}$ In a recent survey of the Scoliosis Research Society members, approximately $80 \%$ of respondents reported routine use of EP monitoring during spinal deformity surgery. ${ }^{8}$ These results are supported by another survey of 27 institutes from UK, Ireland, Australia, and New Zealand, which reported routine use of EP monitoring in all pediatric scoliosis surgeries. ${ }^{14}$ The Scoliosis Research Society has recommended utilization of EP monitoring in spinal deformity surgery as the standard of care when the spinal cord is at risk. ${ }^{8}$ In contrast, the American Association of Neurological Surgeons and Congress of Neurological Surgeons do not support the use of EP monitoring as a therapeutic tool or standard of care in spine surgery, but do support the use of EP monitoring as a diagnostic adjunct to assess perioperative neurologic integrity. ${ }^{15}$ These conflicting opinions pertaining the utility of EP monitoring in scoliosis surgery likely reflects the lack of high-quality evidence upon which to inform clinical decision-making.

The obvious solution to resolve this equipoise is to conduct a well-designed prospective randomizedcontrolled study. Nevertheless, because of the widespread adoption over the past few decades and the perceived benefits of EP monitoring in clinical practice, such a trial would be difficult to conduct in some settings, given the ethical challenges of recruiting and randomizing patients to unmonitored surgeries. ${ }^{5}$ In addition, the overall incidence of neurologic injury of scoliosis surgery has been decreasing over the past few decades, from $0.59 \%$ in 
2004-2007 to $0.39 \%$ in $2013-2016 .^{2}$ This low event rate would require a very large-scale multicentre clinical trial to determine benefit. As a result of these ethical and practical barriers, such a randomized trial is unlikely to be undertaken to resolve this long-standing controversy. The debate of the value of EP monitoring has further been compounded with the high cost and resource utilization, in light of an uncertain benefit, in some jurisdictions. ${ }^{5}$ Unfortunately, we are unable to undertake valid costeffectiveness analyses as the magnitude of treatment effectiveness remains unknown. ${ }^{5}$

Is there "an end" to justify the means? What is clear is that there are insufficient data to conclude that one neuromonitoring technique is superior to another, and we lack robust evidence that neuromonitoring changes patient outcome, including neurologic complications. Many intraoperative monitors are used in the absence of highquality evidence of benefit, and some continue to be employed to provide additional clinical information in high-risk patients, particularly when the complication is potentially devastating. In addition, the choice of monitor must also incorporate patient comfort and acceptance, practicability, cost, and complications from the technique itself. While acknowledging the limitations of Nassef's study, we might reasonably conclude that migration toward EP monitoring from the Stagnara wake-up test was not associated with worse neurologic outcomes in their study patients. As a result, other institutions might use this information to plan their own migration to EP monitoring, particularly given the benefits of avoiding the risks and technical challenges of performing the Stagnara wake-up test, and higher patient acceptance and comfort. As there is a paucity of data to compare neuromonitoring modalities in scoliosis surgery despite their widespread use, even observational data are valuable. Nevertheless, there remains an unmet need for better quality, randomized evidence to support the widespread and routine use of neuromonitoring for scoliosis surgery.

\section{Neuro-monitorage \\ peropératoire pour la chirurgie de la scoliose : $y$ a-t-il une fin pour justifier les moyens?}

Les lésions neurologiques de la moelle épinière ou des racines nerveuses sont une complication redoutée des chirurgies de scoliose. Heureusement, l'incidence des complications neurologiques a considérablement diminué au cours des trente dernières années, passant de $17 \%$ en $1984^{1}$ à $0,39 \%$ en $2016^{2}$ De telles lésions peuvent être provoquées par une traction aiguë ou par l'ancrage de la moelle épinière pendant la correction de la courbure rachidienne, ou à la suite de lésions directes pendant l'instrumentation du rachis. Le test d'éveil de Stagnara a été décrit pour la première fois en $1973^{3}$ et consiste à réveiller le patient au milieu de la chirurgie afin d'évaluer la fonction peropératoire de la moelle épinière. Cette technique initiale de neuro-monitorage était populaire dans les années 1980 et 1990 et demeure la méthode de référence pour évaluer l'intégrité des structures neurologiques en chirurgie de scoliose. Elle ne nécessite pas non plus d'équipement supplémentaire. Dans la pratique néanmoins, le test d'éveil est souvent difficile à réaliser en toute sécurité et ne fournit, dans le meilleur des cas, qu'une évaluation grossière de la fonction motrice à un seul moment de la chirurgie plutôt que de détecter le moment de l'apparition des lésions. En outre, cette période d'anesthésie plus légère peut se compliquer par la perte des voies respiratoires, de l'accès intraveineux ainsi que par la perte de la stérilité du champ opératoire.

L'utilisation des potentiels évoqués somesthésiques (PES) pour monitorer la fonction de la colonne dorsale en tant qu'évaluation de l'intégrité de la moelle épinière a été introduite en clinique pour la première fois dans les années 1980. Par la suite, les potentiels évoqués moteurs (PEM) ont été ajoutés dans les années 1990 pour monitorer l'intégrité fonctionnelle du tractus corticospinal, améliorant la performance diagnostique globale par rapport aux PES utilisés seuls. ${ }^{4}$ Le rôle potentiel de modalités de neuro-monitorage sophistiquées et évolutives pour atténuer les lésions neurologiques est certes séduisant, mais les données probantes de haute qualité appuyant la capacité de ces techniques de neuro-monitorage à améliorer les pronostics neurologiques font défaut. ${ }^{5}$ Malgré l'absence de données probantes convaincantes, les avantages potentiels du neuro-monitorage ont mené à son adoption généralisée dans de nombreuses régions du monde. À l'heure actuelle, l'utilisation du neuro-monitorage semble être motivée par des contextes médico-légaux locaux et divers facteurs économiques, ce qui a donné lieu à une grande variété de pratiques dans le monde.

Dans ce numéro du Journal, Nassef et coll. rapportent les conclusions d'une étude rétrospective avant-après comparant l'effet du test d'éveil de Stagnara au monitorage des potentiels évoqués (PE) sur les déficits neurologiques postopératoires chez 547 patients adolescents (âgés de 10 à 18 ans) ayant subi une chirurgie de correction de scoliose dans deux établissements canadiens entre 2007 et $2014 .^{6}$ Les deux institutions ont modifié leur technique de monitorage neurologique en faisant la transition du test d'éveil de 
Stagnara au monitorage des PE vers 2009/2010. Dans leur analyse, les auteurs ont constaté que les patients ayant reçu un neuro-monitorage peropératoire étaient moins susceptibles de développer des déficits neurologiques postopératoires comparés à ceux ayant subi un test d'éveil de Stagnara (rapport de cotes, 0,39; intervalle de confiance de $95 \%, 0,18$ à 0,$86 ; P=0,020$ ). Les auteurs concluent que le neuro-monitorage peropératoire multimodal (PES et PEM) est associé à une réduction de l'incidence globale des nouveaux déficits neurologiques, bien qu'ils n'aient pas été en mesure d'ajuster leurs résultats pour tenir compte des variables confondantes. ${ }^{6}$ Étant donné le manque de données probantes décrivant le rôle $\mathrm{du}$ monitorage neurologique et des pronostics neurologiques dans la chirurgie du rachis, il convient de féliciter les auteurs pour avoir entrepris cette étude et partagé leur expérience. Cette étude représente l'une des très rares cohortes dans la littérature dans laquelle une comparaison directe des résultats a été réalisée entre ces deux techniques de monitorage neurologique peropératoire lors d'une chirurgie de scoliose.

Bien que cette étude ajoute à la littérature et à notre compréhension du rôle du neuro-monitorage peropératoire, il est important d'interpréter ces résultats dans le contexte des limites de l'étude. En raison de la taille limitée de l'échantillon et du faible taux d'événements de déficits neurologiques dans cette étude, il était impossible d'ajuster les résultats pour tenir compte des variables confondantes potentielles. Ceci est particulièrement important car les modalités de neuro-monitorage étaient séparées dans le temps, ce qui rend probable une confusion historique. Plus précisément, il est probable qu'une tendance constante d'amélioration générale des devenirs de la chirurgie du rachis ait eu lieu au cours de la période à l'étude. Les temps opératoires plus courts et la réduction des pertes de sang observés dans le groupe plus récent de monitorage des PE soutiennent la notion d'une amélioration des pratiques chirurgicale et anesthésique au fil du temps. Une autre limitation inhérente à cette étude rétrospective réside dans l'absence d'évaluations neurologiques normalisées et le risque de données manquantes.

Les données probantes actuelles appuyant l'utilisation du monitorage des PE en chirurgie de scoliose proviennent en grande partie d'études de cohorte rapportant la précision élevée des tests diagnostiques du monitorage des PE pour détecter les lésions neurologiques. Une revue systématique récente de sept études impliquant 2052 patients a rapporté que les estimations ponctuelles des valeurs prédictives positives et négatives combinées de PES et de PEM combinés pour détecter les lésions neurologiques après une chirurgie de scoliose étaient de 12,2 et 99,8 , respectivement. ${ }^{4}$ Une étude canadienne du Centre hospitalier pour enfants de l'est de l'Ontario avait fait état de valeurs prédictives positives et négatives de 12 et 98,2, respectivement. ${ }^{7}$ La quasi totalité de ces études étaient toutefois de nature rétrospective et, surtout, elles souffraient toutes d'un biais potentiel de classification erronée. ${ }^{5}$ Un biais de classification erronée peut survenir lorsqu'une intervention de sauvetage est généralement utilisée en cas d'identification peropératoire de l'alerte au niveau des PE; ainsi l'intervention de sauvetage (c.-à-d. le traitement) pourrait 'prévenir' le devenir avant l'application d'un test de référence (c.-à-d. un examen neurologique postopératoire) dans ces études. Compte tenu de l'incertitude quant à la façon dont les devenirs sont modifiés par l'intervention de sauvetage, la validité de l'exactitude diagnostique rapportée du monitorage des PE pour détecter les lésions neurologiques lors d'une chirurgie de scoliose reste controversée. Pour compliquer davantage la question, d'autres auteurs ont préconisé le monitorage des PE comme outil thérapeutique pour améliorer les pronostics neurologiques après une chirurgie de scoliose. De nombreux comptes rendus ont mis en évidence des cas de lésions neurologiques peropératoires qui ont été rapidement neutralisées par une intervention précoce à la suite d'une détection plus précoce par monitorage des PE. ${ }^{8,9}$ En outre, plusieurs grandes séries de cas ont rapporté une incidence excessivement basse de déficits neurologiques postopératoires chez les patients recevant un monitorage des PE, soutenant ainsi indirectement un rôle thérapeutique positif pour le monitorage des PE. ${ }^{10-13}$ Le rôle réel du monitorage des PE pour améliorer les devenirs reste mal défini, car aucune étude randomisée contrôlée n'a été réalisée pour montrer l'efficacité du monitorage des PE afin de minimiser les lésions neurologiques en chirurgie de scoliose.

Malgré le manque d'efficacité et l'absence d'une évaluation rigoureuse de l'exactitude diagnostique de ce test, le monitorage des $\mathrm{PE}$ a déjà largement remplacé le test d'éveil de Stagnara comme technique de monitorage neurologique de prédilection en chirurgie de scoliose aux États-Unis et dans de nombreux autres pays. Ce changement s'explique en partie par les risques et les difficultés techniques liés à l'exécution du test d'éveil de Stagnara, et en partie par les résultats perçus de non-infériorité chez les patients suivis par monitorage des PE dans le cadre d'études rétrospectives non randomisées. Un compte rendu s'appuyant sur la base de données de l'Échantillon national d'hospitalisation des États-Unis a révélé que l'utilisation du monitorage des PE en chirurgie du rachis avait presque quadruplé, passant de 31762 cas en 2008 à 125835 cas en $2014 .{ }^{13}$ Dans un récent sondage mené auprès des membres de la Scoliosis Research Society, environ $80 \%$ des répondants ont déclaré avoir utilisé de façon routinière le monitorage des $\mathrm{PE}$ pendant une chirurgie de correction de la colonne vertébrale. ${ }^{8}$ Ces 
résultats sont étayés par une autre enquête menée auprès de 27 instituts du Royaume-Uni, d'Irlande, d'Australie et de Nouvelle-Zélande, qui ont signalé une utilisation systématique du monitorage des PE dans toutes les chirurgies pédiatriques de scoliose. $^{14}$ La Scoliosis Research Society a recommandé l'utilisation du monitorage des PE pour les chirurgies de correction de la colonne vertébrale comme norme de soins lorsque la moelle épinière est à risque. ${ }^{8}$ En revanche, l'American Association of Neurological Surgeons et le Congress of Neurological Surgeons ne soutiennent pas l'utilisation du monitorage des PE comme outil thérapeutique ou norme de soins en chirurgie du rachis, mais appuient l'utilisation du monitorage des PE comme complément diagnostique pour évaluer l'intégrité neurologique périopératoire. ${ }^{15} \mathrm{Ces}$ opinions contradictoires concernant l'utilité du monitorage des PE en chirurgie de scoliose reflètent le manque de données probantes nécessaires à éclairer la prise de décision clinique.

La solution évidente pour résoudre cette incertitude absolue est de mener une étude randomisée contrôlée prospective bien conçue. Néanmoins, en raison de l'adoption généralisée au cours des dernières décennies et des avantages perçus du monitorage des PE dans la pratique clinique, une telle étude serait difficile à réaliser, étant donné les défis éthiques liés au recrutement et à la randomisation de patients dans des chirurgies sans monitorage. 5 De plus, l'incidence globale des lésions neurologiques causées par la chirurgie de scoliose a diminué au cours des dernières décennies, passant de $0,59 \%$ en $2004-2007$ à $0,39 \%$ en $2013-2016 .^{2}$ Ce faible taux de complications nécessiterait un essai clinique multicentrique à très grande échelle pour déceler des avantages. En raison de ces obstacles éthiques et pratiques, il est peu probable qu'un tel essai randomisé soit entrepris pour résoudre cette controverse de longue date. Le débat sur la valeur du monitorage des PE a en outre été aggravé par le coût élevé et l'utilisation des ressources, compte tenu d'un avantage incertain, dans certaines juridictions. ${ }^{5}$ Malheureusement, nous ne sommes pas en mesure d'entreprendre des analyses de rentabilité valides, car l'ampleur de l'efficacité du traitement demeure inconnue. ${ }^{5}$

$\mathrm{Y}$ a-t-il « une fin » pour justifier les moyens? Ce qui est clair, c'est qu'il n'y a pas suffisamment de données pour conclure qu'une technique de neuro-monitorage est supérieure à une autre, et nous manquons de données probantes solides selon lesquelles le neuro-monitorage modifie le pronostic des patients, y compris en matière de complications neurologiques. De nombreux moniteurs peropératoires sont utilisés même en l'absence de données probantes de haute qualité de leurs avantages, et certains continuent d'être utilisés pour fournir des informations cliniques supplémentaires chez les patients à haut risque, en particulier lorsque la complication est potentiellement dévastatrice. En outre, le choix du moniteur doit également tenir compte du confort et de l'acceptation du patient, de l'aspect pratique, du coût et des complications de la technique elle-même. Tout en reconnaissant les limites de l'étude de Nassef, nous pouvons cependant raisonnablement conclure que la migration vers le monitorage des PE du test d'éveil de Stagnara n'a pas été associée à de pires pronostics neurologiques chez les patients de l'étude. En conséquence, d'autres institutions pourraient utiliser ces informations pour planifier leur propre migration vers un monitorage des PE, en particulier étant donné les avantages d'éviter les risques et les défis techniques liés à l'exécution du test d'éveil de Stagnara, ainsi que de l'amélioration de l'acceptation et du confort des patients. Étant donné le manque de données nous permettant de comparer les modalités de neuro-monitorage en chirurgie de scoliose en dépit de leur utilisation répandue, les données observationnelles sont précieuses. Néanmoins, il existe encore un besoin à satisfaire en matière de données probantes randomisées de meilleure qualité appuyant l'utilisation répandue et routinière du neuro-monitorage pour la chirurgie de scoliose.

Déclaration Le Dr Jason Chui n'a pas de déclaration ou de conflit d'intérêt relativement à cet éditorial. Dr Alana Flexman est rédactrice adjointe au Journal canadien d'anesthésie.

\section{Déclaration de financement Aucune.}

Responsabilité éditoriale Cet article a été traité par Dr Philip M. Jones, rédacteur adjoint, Journal canadien d'anesthésie.

Disclosures Dr. Jason Chui has no disclosures or conflicts of interest in connection to this Editorial. Dr. Alana Flexman is an Associate Editor at the Canadian Journal of Anesthesia.

Funding statement None.

Editorial responsibility This submission was handled by Dr. Philip M. Jones, Associate Editor, Canadian Journal of Anesthesia.

\section{References}

1. Wilber RG, Thompson GH, Shaffer JW, Brown RH, Nash CL Jr. Postoperative neurological deficits in segmental spinal instrumentation. A study using spinal cord monitoring. J Bone Joint Surg Am 1984; 66: 1178-87.

2. Kwan KY, Koh $H Y$, Blanke KM, Cheung KM. Complications following surgery for adolescent idiopathic scoliosis over a 13-year period. Bone Joint J 2020; 102-B: 519-23.

3. Vauzelle C, Stagnara P, Jouvinroux $P$. Functional monitoring of spinal cord activity during spinal surgery. Clin Orthop Relat Res 1973; 93: 173-8. 
4. Thirumala PD, Huang J, Thiagarajan K, Cheng H, Balzer J, Crammond DJ. Diagnostic accuracy of combined multimodality somatosensory evoked potential and transcranial motor evoked potential intraoperative monitoring in patients with idiopathic scoliosis. Spine (Phila Pa 1976) 2016; 41: E1177-84.

5. Howick J, Cohen BA, McCulloch P, Thompson M, Skinner SA. Foundations for evidence-based intraoperative neurophysiological monitoring. Clin Neurophysiol 2016; 127: 81-90.

6. Nassef $M$, Splinter $W$, Lidster $N$, et al. Intraoperative neurophysiologic monitoring in idiopathic scoliosis surgery: a retrospective observational study of new neurologic deficits. Can J Anesth 2021. DOI: https://doi.org/10.1007/s12630-020-018989.

7. Neira VM, Ghaffari K, Bulusu S, et al. Diagnostic accuracy of neuromonitoring for identification of new neurologic deficits in pediatric spinal fusion surgery. Anesth Analg 2016; 123: 1556-66.

8. Halsey MF, Myung KS, Ghag A, Vitale MG, Newton PO, de Kleuver $M$. Neurophysiological monitoring of spinal cord function during spinal deformity surgery: 2020 SRS neuromonitoring information statement. Spine Deform 2020; 8: 591-6.

9. Fehlings MG, Brodke DS, Norvell DC, Dettori JR. The evidence for intraoperative neurophysiological monitoring in spine surgery: does it make a difference? Spine (Phila Pa 1976) 2010; 35(9 Suppl): S37-46.

10. Nuwer MR, Dawson EG, Carlson LG, Kanim LE, Sherman JE. Somatosensory evoked potential spinal cord monitoring reduces neurologic deficits after scoliosis surgery: results of a large multicenter survey. Electroencephalogr Clin Neurophysiol 1995; 96: 6-11.

11. Hamilton DK, Smith JS, Sansur CA, et al. Rates of new neurological deficit associated with spine surgery based on 108,419 procedures: a report of the scoliosis research society morbidity and mortality committee. Spine (Phila Pa 1976) 2011; 36: 1218-28.

12. Zuccaro M, Zuccaro J, Samdani AF, Pahys JM, Hwang SW. Intraoperative neuromonitoring alerts in a pediatric deformity center. Neurosurgical Focus 2017. DOI: https://doi.org/10.3171/ 2017.7.FOCUS17364.

13. George J, Das S, Egger AC, Chambers RC, Kuivila TE, Goodwin $R C$. Influence of intraoperative neuromonitoring on the outcomes of surgeries for pediatric scoliosis in the United States. Spine Deform 2019; 7: 27-32.

14. Palmer GM, Pirakalathanan P, Skinner AV. A multi-centre multi-national survey of anaesthetists regarding the range of anaesthetic and surgical practices for paediatric scoliosis surgery. Anaesth Intensive Care 2010; 38: 1077-84.

15. Hadley MN, Shank $C D$, Rozzelle CJ, Walters BC. Guidelines for the use of electrophysiological monitoring for surgery of the human spinal column and spinal cord. Neurosurgery 2017; 81: 713-32.

Publisher's Note Springer Nature remains neutral with regard to jurisdictional claims in published maps and institutional affiliations. 